\title{
Eating Behavior and Body Mass Index Status of Senior Regular Undergraduate Students of Addis Ababa University
}

\author{
Tefera Tezera Negera ${ }^{1, *}$, Jemal Haidar Ali $^{2}$ \\ ${ }^{1}$ School of Commerce, Addis Ababa University, Addis Ababa, Ethiopia \\ ${ }^{2}$ Department of Reproductive Health and Health Service Management, School of Public Health, Addis Ababa University, Addis Ababa, \\ Ethiopia
}

\author{
Email address: \\ tefera_tezera@yahoo.com (T. T. Negera),hjemal@gmail.com (J. H. Ali) \\ ${ }^{*}$ Corresponding author
}

\section{To cite this article:}

Tefera Tezera Negera, Jemal Haidar Ali. Eating Behavior and Body Mass Index Status of Senior Regular Undergraduate Students of Addis Ababa University. Science Journal of Public Health. Vol. 4, No. 2, 2016, pp. 107-116. doi: 10.11648/j.sjph.20160402.15

Received: February 9, 2016; Accepted: February 24, 2016; Published: March 12, 2016

\begin{abstract}
The purpose of this study is to assess eating behavior and Body Mass Index status of Senior Regular Undergraduate students of Addis Ababa University. Institutional-based cross-sectional study was conducted among 774 sampled regular senior undergraduate students from three conveniently selected campuses of Addis Ababa University namely the main Campus, Technology and Science faculties. A pre-tested structured and semi structured questionnaires was used to collect the socioeconomic, demographic and eating behavior of the participants. This study highlights the presence of unhealthy eating behaviors attitude and a high prevalence of underweight among university students. It is concluded that promoting healthy eating behavior among young adults is crucial to achieve a healthy nutritional status.
\end{abstract}

Keywords: Eating Behavior, BMI, University Students, Addis Ababa

\section{Background}

The transition of young people from high school to university has many health implications and it is a time of increased responsibility for food choices and practices. They usually tend to engage in problematic eating behaviors that includes unhealthy dieting, high intake of fast food, skipping breakfast, insufficient physical activity, low intake of fruits and vegetables, and minimal consumption of dairy products and thus their nutritional knowledge and food consumption patterns have received global attention [1-4]. During the transition from secondary school to university, students need to adapt to a new environment. When students fail to adapt adequately this could have negative consequences towards their health behaviors and subsequent weight status. Eating behavior (next to physical activity and sedentary behavior) is an important factor influencing students' weight [5]. In addition to rapid changes in physical growth and psychosocial development which have placed them as nutritionally vulnerable groups with poor eating habits, environmental factors also contribute to adoption of unhealthy eating habits [6-8].

Many University Students may be overweight, underweight or obese due to socio demographic and socio economic factors, dietary habits, nutritional knowledge and decrease physical activity. The selection of unhealthy food and high cost of healthy foods may have a negative impact on university student's eating behaviors which requires the attention of the university management [9].

Evidence related to eating habits in relation to BMI status in our country particularly among university students is scare indicating a gap in this regard. It is thus important to uncover the important relevant information and underlines the need for mapping out the magnitude of the problem and avail evidence based information within the higher education institute for appropriate intervention. With this background information, the present study investigated the eating behaviors of university students in relation to their BMI status and identifies the determinant factors for some program initiatives. 


\section{Methodology}

Across-sectional study design was conducted from June2014 to April 2015 among Addis Ababa University (AAU) undergraduate senior regular students with various ethnic populations. The university is the oldest university in the country and located in the capital city of Ethiopia. It has eight multidisciplinary campuses and located in the capital city as well $45 \mathrm{~km}$ South East called Bishofitu and $112 \mathrm{~km}$ north called Fiche campuses. Of the eight campuses, three campuses namely Technology, Natural Sciences and social sciences were conveniently selected because of high number of students representations of the country, accessibility, cost and time. According to the recent data obtained from AAU, 20,120 students were registered in the academic calendar of 2014/15 in the Undergraduate regular program. [10].

\subsection{Ethical Considerations}

Ethical approval was obtained from the School of Public Health Research Ethics Review Committee of Addis Ababa University, College of Health Sciences. Permission was also secured from the Addis Ababa University Students Service Director. Informed written consent was obtained from each subject for their participation and the right to withdraw from the study at any time was also communicated to all of them. Anonymity and confidentiality of the information was assured and privacy of each respondent was maintained throughout the data collection process. All malnourished students were counseled on the importance healthy nutritional behavior.

\subsection{Sample Size Determination}

The required sample size was determined using the magnitude of underweight of $35.6 \%$ [11] and overweight of $9.4 \%$ [12] in selected school communities in the country with level of significance $=0.05$; Marginal of error $=5 \%$; design effect of 2 and non-response rate $=10 \%$. Two different sample sizes of 774 and 287 were estimated for underweight and overweight, respectively. Considering the fact that the prevalence of underweight among university students could give us a logical estimate of the total number of the subjects to be selected for the study, 774 was taken as our final sample size andallocated proportionally across the three campuses social sciences/main campus $(n=196)$, technology $(n=483)$ and natural sciences $(n=96)$. All second year and above students who were in apparent health and registered for the year 2014/2015 were recruited and included systematically (every 5th) until the required sample size for the respective campuses were reached and interviewed.

\subsection{Data Collection Process}

Data were collected using structured questionnaire prepared in the local language Amharic designed by reviewing pertinent research findings on the issue under caption. The important variables included in the questionnaire were socio-demographic, eating attitude containing 36 items and displayed in Table 6 , sources of meal, weight and height measurements. Three data collectors fluent in the local languages and two supervisors (one Health Officer and one Nurse) with relevant experience were recruited and trained for two days on the method of the data collection. The training addressed issues such as the content of the questionnaire, basic interviewing skills, and filling out of the questionnaire, weight and height measurements. The 36 items referring to eating attitude adopted from Nigerian study [13] and were measured using a 4-point Likert scale ranging from rarely/never $=1$; sometimes $=2$; often $=3$ and always $=4$ for all the items and were collected based on participants' selfreported answers in response to the specific questions. All the respondents were interviewed on a one-to-one basis by the PA. The interviews lasted for 25-30 minutes and were conducted during their non-class hours.

\subsection{Anthropometric Measurements}

A digital bathroom scale was used to obtain weight. Prior to each weighing, the scale was adjusted to zero reading to enhance validity. Each participant was weighed twice to improve the accuracy and reliability of measurement; the mean value to the nearest $0.1 \mathrm{~kg}$, as recommended by the World Health Organization was recorded. A standardized measuring board with a fixed head rest and a moveable foot piece e was used to determine height. Height was measured without shoes following standard procedures with the head in upright position and the body firmly stretched and resting on the board.

\subsection{Data Quality Management}

The quality was observed at different levels and included the followings: due emphasis was given to questionnaire designing to capture the objectives of the study, logically sequenced, free of scientific terms and non-leading structured questionnaire and pre-tested before the actual data collection. The data collectors and supervisors were trained on the objective of the study, contents of the questionnaires and how to maintain confidentiality and privacy of the study subjects. The collected data were checked by the principal investigator on daily basis for any incompleteness and/or consistency and timely action was made.

\subsection{Statistical Analysis}

Data were checked for completeness, coded, and entered using Epi-Info version 3.5.4 and then exported to SPSS version 21 for cleaning and analysis. Weight and height of the students were converted to body mass index $\left(\mathrm{BMI}=\mathrm{Kg} / \mathrm{m}^{2}\right)$ as recommended by the World Health Organization and were classified as normal (BMI between 18.5 and $24.9 \mathrm{Kg} / \mathrm{m}^{2}$ ), underweight $\left(\mathrm{BMI}<18.5 \mathrm{Kg} / \mathrm{m}^{2}\right)$ and overweight $(\mathrm{BMI}>25.0$ $\mathrm{Kg} / \mathrm{m} 2$ ). The results are presented in percentages and graphs where appropriate. Binary logistic regression was employed 
to examine the associations between socio-demographic variables and BMI status. To ascertain the association between the dependent variables and the explanatory variables, simultaneously controlling for the aforementioned explanatory variables, (all socio-demographic characteristics and other covariates associated in bivariate with $\mathrm{p}<0.2$ were used and entered) stepwise logistic regression was applied and adjusted odds ratios (AORs) and confidence intervals $(95 \% \mathrm{CI})$ were calculated. In all analyses, $\mathrm{P}<0.05$ was considered to be statistically significant. The 36 items assessing the eating attitudes were evaluated and the means were compared between normal, underweight and overweight/obesity status, respectively.

\section{Result}

A total of 774 respondents participated with a response rate of $97.8 \%$. Out of the 757 students, $614(81.1 \%)$ had dormitory, services, 550(72.7\%) were males and 207(27.3\%) females. The mean age (SD) of the respondents was $(21.6 \pm 1.7)$ years. Over two third $(69.9 \%)$ were in the age groups between $18-22$ years. Over half $(57.6 \%)$ of the respondents were between II and III academic years. About two-thirds $(62.2 \%)$ were from Faculty of Technology, 192(25.4\%) from Social Science and 94(12.4\%) from Faculty of Science. Over a quarter $(29.5 \%)$ had no income, $36(4.8 \%)$ were earning monthly income of less than 100Birr, $167(22.1 \%)$ were earning monthly 100 to 499 Birr and
331(43.7\%) were getting 500 Birr and above. Most (67.9\%) of them used cafeteria service. The proportion of students who used cafeteria services from technology, main campus and natural sciences were $57.1 \%, 28.2 \%$ and $14.7 \%$, respectively. Regarding eating place of none cafeteria users, $165(67.9 \%)$ of them consumed elsewhere and less than a third $(32.1 \%)$ prepared their food for themselves. Over a quarter $(28.0 \%)$ of mothers and one third $(35.8 \%)$ of the fathers had completed tertiary education (Table 1).

The mean BMI and year of study of both genders were $\left(19.8 \pm 2.7 \mathrm{~kg} / \mathrm{m}^{2}\right)$ and $(2.4 \pm 1.1$ years $)$, respectively. Nonetheless, when the weight, height and BMI of student were compared between genders, males had significantly higher weight $(p=0.001)$, height $(p=0.001)$ and BMI $(p=0.001)$ than females. Likewise the mean academic year was also higher in males than females though the difference noted was not significantly $(\mathrm{p}=0.42)$ (Table 2$)$.

Figure 1 shows the BMI status of the students by Gender. As shown the overall proportion of Sever thinness (BMI < 16.0), Moderate thinness (BMI $16.0-16.99$ ) and Mild thinness (BMI $17.00-18.49$ ) was $2.2 \%, 7.0 \%$ and $25.1 \%$, respectively. Likewise, the overall overweight (BMI 25.0 29.9) and obese class I (BMI 30.0 -34.99) was also 3.7\% and $0.5 \%$. When the findings are disaggregated by students genders, severe thinness was significantly higher in males than female $(\mathrm{p}=0.03)$. The proportion of overweight was also higher in males than the females though the difference noted was not significantly $(p=0.4)$.

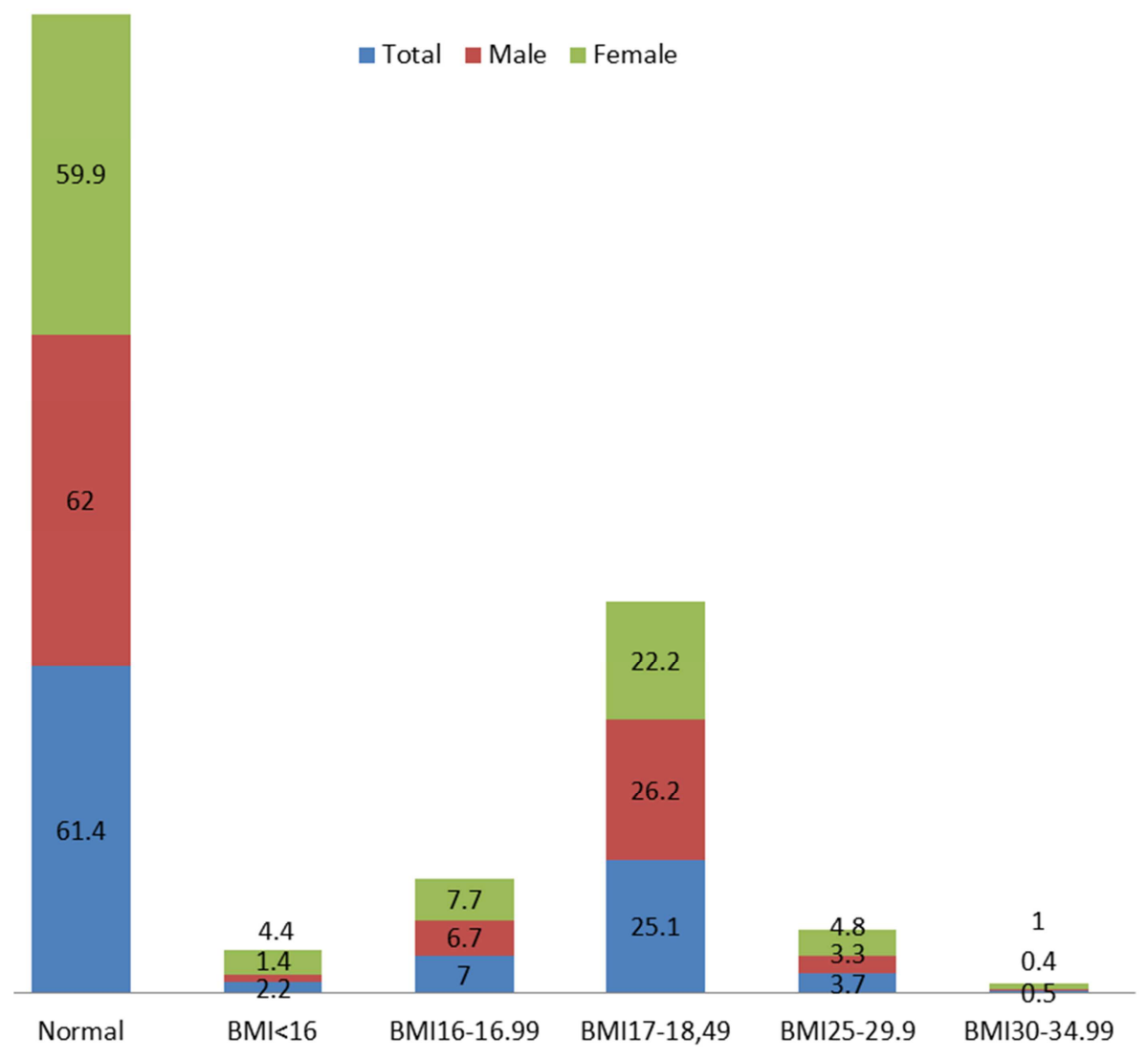

Figure 1. BMI of students by gender, Addis Ababa University, 2015. 
Table 1. Socio-demographic and other related characteristics of students, Addis Ababa university, 2015 $(N=757)$.

\begin{tabular}{|c|c|c|c|}
\hline Variable & Category & Frequency & Percentage \\
\hline \multirow[t]{2}{*}{ Resident area } & In campus & 614 & 81.1 \\
\hline & Outside campus & 143 & 18.9 \\
\hline \multirow[t]{2}{*}{ Sex } & Male & 550 & 72.7 \\
\hline & Female & 207 & 27.3 \\
\hline \multirow[t]{3}{*}{ Age } & $18-22$ & 529 & 69.9 \\
\hline & $23-27$ & 225 & 29.7 \\
\hline & $28-32$ & 3 & 0.4 \\
\hline \multirow[t]{4}{*}{ Year of Training } & II- Year & 208 & 27.5 \\
\hline & III- Year & 228 & 30.1 \\
\hline & IV- Year & 162 & 21.4 \\
\hline & V- Year & 159 & 21.0 \\
\hline \multirow[t]{3}{*}{ Field of study } & Technology & 471 & 62.2 \\
\hline & Social Science & 192 & 25.4 \\
\hline & Science & 94 & 12.4 \\
\hline \multirow[t]{5}{*}{ Monthly income(Birr) } & No income & 323 & 29.5 \\
\hline & Less than 100-299 & 107 & 14.2 \\
\hline & From 300 to 499 & 96 & 12.7 \\
\hline & 500 and above & 246 & 32.5 \\
\hline & Don't now & 85 & 11.1 \\
\hline \multirow[t]{2}{*}{ Used campus cafeteria } & Yes & 514 & 67.9 \\
\hline & No & 243 & 32.1 \\
\hline \multirow[t]{3}{*}{$\begin{array}{l}\text { Distribution by } \\
\text { cafeteria(n=515) }\end{array}$} & Technology & 294 & 57.1 \\
\hline & Main campus & 145 & 28.2 \\
\hline & Science campus & 76 & 14.7 \\
\hline \multirow[t]{2}{*}{$\begin{array}{l}\text { Eating place for none- } \\
\text { café users }(n=243)\end{array}$} & $\begin{array}{l}\text { Home/ prepared } \\
\text { by self }\end{array}$ & 78 & 32.1 \\
\hline & Outside cafeteria & 165 & 67.9 \\
\hline \multirow{4}{*}{$\begin{array}{l}\text { Mother educational } \\
\text { status }\end{array}$} & Non-formal & 148 & 19.5 \\
\hline & $\begin{array}{l}\text { High school or } \\
\text { less }\end{array}$ & 220 & 29.1 \\
\hline & Tertiary Education & 212 & 28.0 \\
\hline & Not educated & 177 & 23.4 \\
\hline \multirow[t]{4}{*}{$\begin{array}{l}\text { Father educational } \\
\text { status }\end{array}$} & Non-formal & 138 & 18.2 \\
\hline & $\begin{array}{l}\text { High school or } \\
\text { less }\end{array}$ & 214 & 28.3 \\
\hline & Tertiary Education & 271 & 35.8 \\
\hline & Not educated & 124 & 17.7 \\
\hline
\end{tabular}

$1 \mathrm{USD}=20.78$ Birr at the time of the study
Table 2. Anthropometric and mean academic year of students by sex, Addis Ababa University, 2015 $(N=757)$.

\begin{tabular}{|c|c|c|c|c|}
\hline Variable & Total $(N=757)$ & $\begin{array}{l}\text { Male } \\
(\mathbf{N}=\mathbf{5 5 0})\end{array}$ & $\begin{array}{l}\text { Female } \\
(\mathrm{N}=\mathbf{2 0 7})\end{array}$ & P. Value \\
\hline BMI ( $( \pm \mathrm{SD})$ & $19.8 \pm 2.7 \mathrm{~kg} / \mathrm{m}^{2}$ & $19.7 \pm 2.6$ & $20.1 \pm 3.1$ & 0.001 \\
\hline $\begin{array}{l}\text { Mean years of } \\
\text { study }( \pm \mathrm{SD})\end{array}$ & $2.4 \pm 1.1$ & $2.5 \pm 1.1$ & $2.0 \pm 1.0$ & 0.42 \\
\hline Weight ( \pm SD) & $56.3 \pm 8.9 \mathrm{~kg}$ & $58.1 \pm 8.5$ & $51.6 \pm 8.4$ & 0.001 \\
\hline Height ( $\pm \mathrm{SD})$ & $1.8 \pm 3.1 \mathrm{mts}$ & $1.86 \pm 3.7$ & $1.6 \pm 0.06$ & 0.001 \\
\hline
\end{tabular}

\subsection{Eating Habits of University Students}

Eating habits of the students by gender is displayed in Table 3. The overall proportion of students taking their meals regularly, breakfast daily, lunch daily, dinner daily was $66.2 \%, 50.4 \%, 88.7 \%$ and $88.0 \%$, respectively. Nonetheless, when consumption was compared between the two genders, the proportion was higher among females than males.

Over half $(53.3 \%)$ of them took their meals 3 times daily and $248(45.1 \%)$ rarely consumed snack and breakfast was the most $(68.4 \%)$ common meal skipped by most of the students with female students being in a better position. The practice of eating vegetables, fruits, fried food, drinking alcohol was rare in both genders. Eating daily with friends and family was mentioned in over a quarter $(29.5 \%)$ of them and the proportion was higher in males $(42.0 \%)$ than females $(29.5 \%)$. The majority $(81.8 \%)$ were aware of the type of food they should eat in order to have balanced nutrition.

\subsection{Association Between Socio Demographic Variables and Underweight}

As shown in Table 4, Using the campus Cafeteria, place of the cafeteria and Eating place of non- café users were significantly associated with the level of underweight. The proportion of underweight was significantly lower among campus cafeteria users $[\mathrm{COR}=0.67 ; 95 \% \mathrm{CI}=0.48$ to 0.94 ] and who consumed their meal from technology faculty cafeteria $[\mathrm{COR}=0.59 ; 95 \% \mathrm{CI}=0.40$ to 0.86$]$ than the referent groups. In contrast the proportion of underweight was higher among those who prepared their food than their counterparts $\quad[\mathrm{COR}=1.82 ; \quad 95 \% \mathrm{CI}=1.03$ to 3.19]. Nonetheless, age, sex, year of study, maternal education, father education, monthly income and resident area were not associated with underweight of the respondents. After controlling for the confounding effect of socio-demographic variables, only for those using the campus Cafeteria, and Eating place of non- café users remained significant. The odds for being underweight was 1.1 times $[\mathrm{AOR}=1.12$; $95 \% \mathrm{CI}=1.23$ to 2.33 ] among café users and among those preparing their food $[\mathrm{AOR}=1.736 ; 95 \% \mathrm{CI}=1.19$ to 2.79$]$ than their referent groups. 
Table 3. Eating habits of students by sex, Addis Ababa University, 2015 ( $N=757)$.

\begin{tabular}{|c|c|c|c|c|}
\hline Eating habits & Category & Total & Male & Female \\
\hline \multirow{2}{*}{ Do you take your meals regularly? } & Always regular & $364(66.2)$ & 99(47.8.) & $463(61.2)$ \\
\hline & Irregular & $186(33.8)$ & $108(52.2)$ & 294(38.8) \\
\hline \multirow{4}{*}{ Do you always take breakfast daily? } & Daily & $277(50.4)$ & $74(35.7)$ & $351(46.4)$ \\
\hline & 3-4 times per week & $167(30.4)$ & $63(30.4)$ & $230(30.4)$ \\
\hline & 1-2 times per week & $29(5.2)$ & $19(9.3)$ & $48(6.3)$ \\
\hline & Rarely & $77(14.0)$ & $51(24.6)$ & $128(16.9)$ \\
\hline \multirow{4}{*}{ Do you always take lunch daily? } & Daily & $488(88.7)$ & $168(81.2)$ & $656(86.7)$ \\
\hline & 3-4 times per week & $43(7.8)$ & $21(10.1)$ & $64(8.5)$ \\
\hline & 1-2 times per week & $4(0.7)$ & $4(1.9)$ & $8(1.1)$ \\
\hline & Rarely & $15(2.8)$ & $14(6.8)$ & $29(3.7)$ \\
\hline \multirow{4}{*}{ Do you always take dinner daily? } & Daily & $484(88.0)$ & $127(61.4)$ & $611(80.7)$ \\
\hline & 3-4 times per week & $48(8.7)$ & $47(22.7)$ & $95(12.5)$ \\
\hline & 1-2 times per week & $6(1.1)$ & $8(3.9)$ & $14(1.8)$ \\
\hline & Rarely & $12(2.2)$ & $25(12.1)$ & $37(4.9)$ \\
\hline \multirow{4}{*}{ How many times do you eat your regular meals? } & Once & $124(22.5)$ & $40(19.3)$ & $164(21.7)$ \\
\hline & Two times & $105(19.1)$ & $54(28.5)$ & 164(21.7) \\
\hline & Three times & 293(53.3) & $99(47.8)$ & $392(51.8)$ \\
\hline & Four times & $28(5.1)$ & $9(4.4)$ & $37(4.8)$ \\
\hline \multirow{4}{*}{$\begin{array}{l}\text { How often do you take snacks apart from regular } \\
\text { meals daily? }\end{array}$} & Daily & $69(12.5)$ & $29(14.50$ & $98(12.9)$ \\
\hline & 3-4 times per week & $122(22.2)$ & $54(26.1)$ & $176(23.3)$ \\
\hline & 1-2 times per week & $111(20.2)$ & $47(22.7)$ & $158(20.9)$ \\
\hline & Rarely & $248(45.1)$ & $77(37.2)$ & $325(42.9)$ \\
\hline \multirow{4}{*}{ How many meals do you usually eat each day? } & One & $42(7.6)$ & $10(4.8)$ & $52(6.9)$ \\
\hline & Two & $117(21.3)$ & $70(33.8)$ & 187(24.7) \\
\hline & Three & $352(64.0)$ & $114(55.1)$ & $466(61.6)$ \\
\hline & Four & $39(7.1)$ & $13(6.3)$ & $52(6.8)$ \\
\hline \multirow{3}{*}{ If you skip a meal, which meal is it usually? } & Breakfast & $376(68.4)$ & $131(63.3)$ & $507(67.0)$ \\
\hline & Lunch & $87(15.8)$ & $29(14.0)$ & $116(15.3)$ \\
\hline & Dinner & $87(15.8)$ & $47(22.7)$ & 134(17.7) \\
\hline \multirow{4}{*}{$\begin{array}{l}\text { How often do you eat green, red or yellow colour } \\
\text { vegetables daily? }\end{array}$} & Daily & $11(2.0)$ & $7(3.4)$ & $18(2.4)$ \\
\hline & 3-4 times per week & $39(7.1)$ & $36(17.4)$ & $75(9.9)$ \\
\hline & 1-2 times per week & $227(41.3)$ & $82(39.6)$ & $309(40.8)$ \\
\hline & Rarely & 273(49.6) & $82(39.6)$ & $355(46.9)$ \\
\hline \multirow{4}{*}{ How often do you eat fruits daily? } & Daily & $10(1.8)$ & $6(2.9)$ & $16(2.1)$ \\
\hline & 3-4 times per week & $26(4.7)$ & $21(10.0)$ & $47(6.2)$ \\
\hline & 1-2 times per week & $156(28.4)$ & $72(34.0)$ & $228(30.1)$ \\
\hline & Rarely & $358(65.1)$ & $108(52.2)$ & $466(61.6)$ \\
\hline \multirow{4}{*}{ How often do you eat fried food? } & Daily & $44(8.0)$ & $23(11.2)$ & $67(8.9)$ \\
\hline & 3-4 times per week & $87(15.8)$ & $45(21.7)$ & 132(17.4) \\
\hline & 1-2 times per week & $132(24.0)$ & $59(28.5)$ & 191(25.2) \\
\hline & Rarely & $287(52.2)$ & $80(38.6)$ & $367(48.5)$ \\
\hline \multirow{3}{*}{ How often do you take alcohol? } & Daily & $10(1.8)$ & $6(2.9)$ & $16(2.1)$ \\
\hline & 2-3times per week & $43(7.8)$ & $7(3.4)$ & $50(6.6)$ \\
\hline & Rarely & 497(90.4) & 194(93.7) & $691(91.3)$ \\
\hline \multirow{4}{*}{$\begin{array}{l}\text { How often do you eat with friends and family } \\
\text { daily? }\end{array}$} & Daily. & $136(24.7)$ & $87(42.0)$ & $223(29.5)$ \\
\hline & 3-4 times per week & $114(20.7)$ & $34(16.4)$ & $148(19.6)$ \\
\hline & 1-2 times per week & $149(27.1)$ & $55(26.6)$ & 204(26.9) \\
\hline & Always alone & $151(27.5)$ & $31(15.0)$ & $182(24.0)$ \\
\hline \multirow{3}{*}{$\begin{array}{l}\text { What type of food do you think you should eat to } \\
\text { have a balanced nutrition? }\end{array}$} & Mainly meat & $34(6.2)$ & $8(3.9)$ & $42(5.5)$ \\
\hline & Mainly vegetable & $66(12.0)$ & $44(21.3)$ & $110(14.5)$ \\
\hline & Variety of food & $450(81.8)$ & $155(74.8)$ & $605(80.0)$ \\
\hline
\end{tabular}


Table 4. Bivariate and logistic regression analysis of socioeconomic factors associated with participants underweight, Addis Ababa University, 2015.

\begin{tabular}{|c|c|c|c|c|}
\hline Variable & Category & Underweight & $\operatorname{COR}(95 \% \mathrm{CI})$ & $\operatorname{AOR}(95 \% \mathrm{CI})$ \\
\hline \multirow{2}{*}{ Sex } & Male & $189(72.7)$ & $1.033(0.734,1.454)$ & $1.315(0.842,2.054)$ \\
\hline & Female & $71(27.3)$ & 1.0 & 1.0 \\
\hline \multirow{3}{*}{ Age } & $18-22$ & 181(69.6) & ---- & ------- \\
\hline & $23-27$ & $79(30.4)$ & ----- & ------- \\
\hline & $28-32$ & $0(0)$ & 1.0 & 1.0 \\
\hline \multirow{4}{*}{ Year of Training } & II- year & $71(27.3)$ & $1.072(0.693,1.660)$ & $0.943(0.534,1.667)$ \\
\hline & III- Year & $69(26.6)$ & $1.287(0.834,1.986)$ & $1.282(0.753,2.183)$ \\
\hline & IV- Year & $62(23.8)$ & $0.935(0.593,1.476)$ & $0.856(0.513,1.427)$ \\
\hline & V- Year & $58(22.3)$ & 1.0 & 1.0 \\
\hline \multirow{3}{*}{ Field of study } & Technology & $172(66.2)$ & $0.777(0.480,1.257)$ & $0.682(0.206,2.255)$ \\
\hline & Social Science & $59(22.7)$ & $0.991(0.557,1.701)$ & $1.595(0.386,6.579)$ \\
\hline & Science & $29(11.1)$ & 1.0 & 1.0 \\
\hline \multirow{4}{*}{ Mothers education } & Non- formal & $44(16.9)$ & $1.396(0.874,2.229)$ & $1.599(0.935,2.735)$ \\
\hline & Secondary & $82(31.5)$ & $0.946(0.626,1.429)$ & $0.915(0.531,1.579)$ \\
\hline & Tertiary 1 & $67(25.8)$ & $1.231(0.807,1.880)$ & $1.083(0.597,1.965)$ \\
\hline & Not educated & $67(25.8)$ & 1.0 & 1.0 \\
\hline \multirow{4}{*}{ Fathers education } & Non formal & $49(18.8)$ & $0.918(0.556,1.517)$ & $0.789(0.443,1.406)$ \\
\hline & Secondary & $78(30.1)$ & $0.816(0.516,1.290)$ & $0.694(0.391,1.233)$ \\
\hline & Tertiary & $88(33.8)$ & $0.987(0.634,1.537)$ & $0.763(0.408,1.426)$ \\
\hline & Not Educates & $45(17.3)$ & 1.0 & 1.0 \\
\hline \multirow{6}{*}{ Monthly income (ETB) } & No income & $75(28.8)$ & $1.245(0.736,2.107)$ & $1.189(0.685,2.066)$ \\
\hline & Less than 100 & $15(5.8)$ & $0.784(0.346,1.775)$ & $0.742(0.314,1.753)$ \\
\hline & From 100 to 299 & $32(12.3)$ & $0.796(0.417,1.518)$ & $0.838(0.422,1.664)$ \\
\hline & From 300 to 499 & $35(13.5)$ & $1.064(0.576,1.963)$ & $1.086(0.567,2.082)$ \\
\hline & 500 and above & $7(27.3)$ & $1.462(0.576,1.963)$ & $1.529(0.858,2.725)$ \\
\hline & I don't now & $32(12.3)$ & 1.0 & 1.0 \\
\hline \multirow{2}{*}{ Use campus Cafeteria } & Yes & $193(74.2)$ & $0.675(0.481,0.941)^{*}$ & $1.115(1.245,2.329)^{*}$ \\
\hline & No & $67(25.8)$ & 1.0 & 1.0 \\
\hline \multirow{3}{*}{$\begin{array}{l}\text { Place of cafeteria of the } \\
\text { campuses }\end{array}$} & Technology & $119(45.8)$ & $0.593(0.409,0.860)^{*}$ & -------- \\
\hline & Main campus & $51(19.6)$ & $0.729(0.466,1.141)$ & ------- \\
\hline & Science campus & $24(9.2)$ & 1.0 & 1.0 \\
\hline \multirow{3}{*}{$\begin{array}{l}\text { Eating place of non- café } \\
\text { users }\end{array}$} & Self Prepared & $18(6.9)$ & $1.816(1.032,3.197)^{*}$ & $1.736(1.194,2.798)^{*}$ \\
\hline & Outside cafeteria & $45(17.3)$ & $1.285(0.862,1.916)$ & $0.535(0.156,1.836)$ \\
\hline & Other & $4(1.5)$ & 1.0 & 1.0 \\
\hline \multirow{2}{*}{ Reside in campus } & yes & $222(85.4)$ & $0.676(0.448,1.020)$ & $0.653(0.319,1.771)$ \\
\hline & No & $38(14.6)$ & 1.0 & 1.0 \\
\hline
\end{tabular}

I USD $=20.87 \mathrm{ETB}$ at the time of the study

\subsection{Association Between Socio-demographic Variables and Overweight}

Likewise, overweight was significantly associated with the year of study[COR $=0.59 ; 95 \% \mathrm{CI}=0.20$ to 0.77$]$, father education $[\mathrm{COR}=4.59 ; 95 \% \mathrm{CI}=1.01$ to 20.81$]$, monthly income $[\mathrm{COR}=4.76 ; 95 \% \mathrm{CI}=1.05$ to 21.64$]$, campus cafeteria users $[\mathrm{COR}=2.49 ; 95 \% \mathrm{CI}=1.21$ to 5.15$]$, place of cafeteria of the campuses [COR $=3.20 ; 95 \% \mathrm{CI}=1.24$ to 8.28 ] and eating place of none cafeteria users[COR $=0.29$; $95 \% \mathrm{CI}=0.11$ to 0.74$]$. The odds of having overweight was $41.0 \%$ less likely among year four students and $71.0 \%$ among those eating outside café than the referent groups. While it was 4.6 times higher among who had no formal education, 4.76 times more likely among those earning below 100ETB, 2.5 times more among café users and 3.2 times more likely among campus users than the referent groups. Nevertheless, after controlling for the confounding effect of socio-demographic variables, the association remained significant for non- café users $[\mathrm{AOR}=2.844$; 95\% CI (1.02 to 7.89)]. The odds of being overweight were 1.1 times more among non-café users than their referent groups (Table 5).

\subsection{Eating Attitude of Respondents}

The four-item scale constructed to assess the extent of the variation of BMI status of participants in relation to eating habits is displayed in Table 6 . The highest mean scores for underweight students were found on items 7, 29 and 33. For normal weight student, item 19, 7, 29 and 33 ranked highest while for the overweight students, items 21 and 33 and 33 had the highest mean scores. Whereas for obesity, the highest mean scores items 2, 6, 7, 11, 13, 14, 29, 30 and all raked highest. Although, the mean scores showed some variation with BMI, significant differences between normal and overweight status were observed in only in items 2 (I seriously would like to learn cooking), 4 (I eat precooked food, instant and frozen 
products, and delivery foods), 18 (I try to eat at the same time every day), 24 (I want to lose weight just by eating a well- balanced diet) and 36 (I like to strengthen relationships with others by eating together).

Table 5. Bivariate and logistic regression analysis of socioeconomic factors associated with participants overweight, Addis Ababa University, 2015.

\begin{tabular}{|c|c|c|c|c|}
\hline Variables & Category & Over-Weight & $\operatorname{COR}(95 \% \mathrm{CI})$ & $\operatorname{AOR}(95 \% \mathrm{CI})$ \\
\hline \multirow{2}{*}{ Sex } & Male & $20(62.5)$ & 1.0 & 1.0 \\
\hline & Female & $12(37.5)$ & $0.606(0.288,1.276)$ & $0.949(0.342,2.488)$ \\
\hline \multirow{3}{*}{ Age } & $18-22$ & $24(75.0)$ & 1.0 & 1.0 \\
\hline & $23-27$ & $7(29.9)$ & $0.680(0.286,16.150)$ & $0.819(0.331,2.026)$ \\
\hline & $28-32$ & $1(3.1)$ & $6.750(0.591,77.134)$ & $9.498(0.446,20.330)$ \\
\hline \multirow{3}{*}{ Year of study } & II-year & $11(34.4)$ & 1.0 & 1.0 \\
\hline & III-year & $12(37.5)$ & $0.935(0.399,2.192)$ & $1.197(0.458,3.124)$ \\
\hline & V-year & $5(15.6)$ & $0.597(0.201,0.774)^{*}$ & $0.931(0.260,3.333)$ \\
\hline \multirow{3}{*}{ Field of study } & Technology & $18(56.3)$ & 1.0 & 1.0 \\
\hline & Social Science & $10(31.2)$ & $1.269(0.569,2.829)$ & $1.513(0.457,5.009)$ \\
\hline & Science & $4(12.5)$ & $1.024(0.335,3.132)$ & $0.684(0.072,6.508)$ \\
\hline \multirow{4}{*}{ Mother education } & Non-formal & $5(15.6)$ & 1.0 & 1.0 \\
\hline & Secondary & $13(40.6)$ & $2.059(0.710,5.971)$ & $1.162(0.358,3.769)$ \\
\hline & Tertiary & $12(37.5)$ & $1.786(0.610,5.235)$ & $1.020(0.278,3.740)$ \\
\hline & Not-educated & $2(6.3)$ & $0.367(0.070,1.933)$ & $0.377(0.061,2.343)$ \\
\hline \multirow{3}{*}{ Father education } & Secondary & $13(40.7)$ & $4.598(1.012,20.891)^{*}$ & $3.436(0.695,16.984)$ \\
\hline & Tertiary & $15(46.9)$ & $3.884(0.868,17.371)$ & $2.514(0.461,13.716)$ \\
\hline & Not-educated & $2(6.2)$ & $1.000(0.138,7.260)$ & $1.552(0.188,12.834)$ \\
\hline \multirow{5}{*}{ Monthly income in (ETB) } & No income & $5(15.6)$ & 1.0 & 1.0 \\
\hline & Less than 100 & $3(9.4)$ & $4.767(1.050,21.641)^{*}$ & $4.557(0.908,22.863)$ \\
\hline & From 100to 299 & $0(0.0)$ & $2.007(0.520,7.743)$ & $1.852(0.464,7.397)$ \\
\hline & From 300to 499 & $4(12.5)$ & $2.878(1.028,8.056)^{*}$ & $2.116(0.710,6.309)$ \\
\hline & I do not know & $16(50.0)$ & $2.335(0.603,9.044)$ & $2.227(0.563,8.812)$ \\
\hline \multirow{2}{*}{ Use campus Cafeteria } & Yes & $14(43.8)$ & 1.0 & 1.0 \\
\hline & No & $18(56.2)$ & $2.498(1.211,5.155)^{*}$ & $2.844(1.025,7.891)^{*}$ \\
\hline \multirow{3}{*}{$\begin{array}{l}\text { Place of cafeteria of the } \\
\text { campuses }\end{array}$} & Technology & $6(18.8)$ & 1.0 & 1.0 \\
\hline & Main campus & $5(15.6)$ & $1.724(0.416,7.148)$ & $3.213(0.226,45.612)$ \\
\hline & Science campus & $3(9.4)$ & $3.209(1.242,8.289)^{*}$ & $2.309(0.586,9.103)$ \\
\hline \multirow{2}{*}{$\begin{array}{l}\text { Eating place of none } \\
\text { cafeteria users }\end{array}$} & Self Prepared & $8(25.0)$ & 1.0 & 1.0 \\
\hline & Outside cafeteria & $8(25.0)$ & $0.929(0.177,4.874)$ & $0.490(0.169,1.417)$ \\
\hline Resides in campus & No & $11(34.4)$ & $2.067(0.963,4.437)$ & $0.916(0.269,3.111)$ \\
\hline
\end{tabular}

Table 6. Item mean for eating attitudes in relation to BMI status of participants, Addis Ababa University, 2015.

\begin{tabular}{|c|c|c|c|c|c|c|}
\hline & Item & $\begin{array}{l}\text { Under-weight } \\
(\mathrm{N}=260)\end{array}$ & $\begin{array}{l}\text { Normal weight } \\
(\mathrm{N}=465)\end{array}$ & $\begin{array}{l}\text { Over-weight } \\
(\mathrm{N}=\mathbf{2 8})\end{array}$ & $\begin{array}{l}\text { Obesity } \\
(\mathrm{N}=4)\end{array}$ & P-Value \\
\hline 1 & I try to eat a well -balanced diet. & 2.14 & 2.31 & 2.4 & 2 & 0.097 \\
\hline 2 & I seriously would like to learn cooking. & 2.3 & 2.4 & 3 & 3.5 & $0.016^{*}$ \\
\hline 3 & I check for food additive, food coloring, etc in my food. & 1.96 & 1.93 & 2.17 & 2.75 & 0.499 \\
\hline 4 & $\begin{array}{l}\text { I eat precooked food, instant and frozen products, and delivery } \\
\text { foods (e.g, pizza). }\end{array}$ & 1.78 & 2.02 & 2.14 & 2.25 & 0.006 \\
\hline 5 & $\begin{array}{l}\text { I take nutritional supplements like vitamin tablets or similar } \\
\text { products. }\end{array}$ & 1.75 & 1.76 & 1.46 & 2 & 0.686 \\
\hline 6 & The amount of my food intake varies depending on my mood. & 2.7 & 2.6 & 2.46 & 3.5 & 0.366 \\
\hline 7 & Practicing healthy eating behavior is important to me. & 3.04 & 2.92 & 2.92 & 3.5 & 0.616 \\
\hline 8 & I would like people to commend my cooking. & 2.21 & 2.18 & 2 & 3 & 0.639 \\
\hline 9 & I buy natural foods and organic vegetables even if they cost more. & 1.9 & 1.99 & 2 & 3 & 0.639 \\
\hline 10 & I try not to eat too much. & 2.7 & 2.68 & 2.46 & 2.75 & 0.131 \\
\hline 11 & Fast food is delicious and convenient. & 2.78 & 2.72 & 2.6 & 3.5 & 0.663 \\
\hline 12 & I highly value precooked dishes in the supermarket. & 1.84 & 1.87 & 1.78 & 1.5 & 0.804 \\
\hline 15 & I feel uneasy about trusting important foods. & 2.14 & 2.26 & 2.32 & 2.5 & 0.208 \\
\hline 16 & I want to save money on food and spend it on other things. & 1.97 & 2.08 & 2.07 & 2 & 0.197 \\
\hline 17 & I try to eat a variety of foods. & 2.42 & 2.56 & 2.57 & 2.75 & 0.552 \\
\hline 18 & I try to eat at the same time every day. & 2.38 & 2.53 & 2.57 & 2.75 & $0.03 *$ \\
\hline 19 & I try to enjoy eating my meals. & 2.75 & 2.9 & 2.85 & 3 & 0.173 \\
\hline 20 & I think there are many tasty instant noodle soups. & 2.07 & 2.2 & 2.32 & 2.5 & 0.778 \\
\hline 21 & I would like to know more about food nutrients and what functions & 2.86 & 2.85 & 2.96 & 3.25 & 0.6 \\
\hline
\end{tabular}




\begin{tabular}{|c|c|c|c|c|c|c|}
\hline & Item & $\begin{array}{l}\text { Under-weight } \\
(\mathrm{N}=\mathbf{2 6 0})\end{array}$ & $\begin{array}{l}\text { Normal weight } \\
(\mathrm{N}=\mathbf{4 6 5})\end{array}$ & $\begin{array}{l}\text { Over-weight } \\
(\mathrm{N}=28)\end{array}$ & $\begin{array}{l}\text { Obesity } \\
(\mathrm{N}=4)\end{array}$ & P-Value \\
\hline & they have. & & & & & \\
\hline 22 & I do not care what I eat as long as it fills my stomach. & 1.8 & 2.03 & 1.85 & 2.25 & 0.166 \\
\hline 23 & $\begin{array}{l}\text { I want to buy and try out new food products as soon as they are } \\
\text { released. }\end{array}$ & 1.79 & 1.95 & 1.64 & 2 & 0.278 \\
\hline 24 & I want to lose weight just by eating a well- balanced diet. & 1.74 & 2.03 & 2.5 & 2.75 & $0.001 *$ \\
\hline 25 & I always drink vitamins drinks. & 1.69 & 1.76 & 1.42 & 3 & 0.069 \\
\hline 26 & I don't mind eating the same things every day. & 2.26 & 2.13 & 2.32 & 3.25 & 0.225 \\
\hline 27 & I only want to eat my favorite dishes. & 2.6 & 2.48 & 2.35 & 2.25 & 0.248 \\
\hline 28 & I dislike cooking and cleaning up afterwards. & 1.91 & 1.96 & 1.78 & 2.25 & 0.853 \\
\hline 29 & When food taste good, I eat more than usual. & 2.96 & 3 & 2.67 & 3.5 & 0.282 \\
\hline 30 & I worry about calories when eating. & 2 & 2.03 & 2.03 & 3.5 & 0.234 \\
\hline 31 & My eating habits are normal. & 2.64 & 2.8 & 2.53 & 3 & 0.664 \\
\hline 32 & It’s OK not to eat. & 1.8 & 1.73 & 1.78 & 2.5 & 0.099 \\
\hline 33 & I like to eat. & 3.2 & 3.19 & 3.14 & 3.25 & 0.908 \\
\hline 34 & I feel like eating when I am in a bad mood. & 1.72 & 1.97 & 1.78 & 1.75 & 0.248 \\
\hline 35 & When I see a person eating, I want to eat as well. & 2.25 & 2.35 & 2.14 & 2 & 0.282 \\
\hline 36 & I like to strengthen relationships with other by eating together. & 2.76 & 2.76 & 2.53 & 1.25 & $0.033^{*}$ \\
\hline
\end{tabular}

\section{Discussion}

In this study, the distribution of the sampled students' eating attitude and behavior was described in relation to their BMI status and was categorized into underweight, normal, overweight/obesity, respectively. Based on this assumption, the overall prevalence of underweight, overweight and obesity findings found in this study were closer to the Gondar study underweight $(34.3 \% \mathrm{vs} 35.6 \%)$, overweight $(3.7 \%$ vs $3.9 \%)$ and obesity $(0.5 \%$ vs $0.7 \%)$ [7]. When compared with the Sudanese study, the present overweight finding was lower (3.7\% vs $14.8 \%$ ) and the observed difference is attributed to the high energy dense foods and frequently eating habits among the students in Sudan [14].

Breakfast is a regularly missed meal among most university students due to time constraints [15]. Similarly, in the present study, it was frequently skipped meal among both male and female students and appeared to be higher $(68.4 \%$ vs $42.3 \%$ ) when compared with the Sidama study in southern Ethiopia [16]. Whereas when the present findings is compared with Ankara University students, Turkey, the figure for skipping meals among Ankara students is higher $(68,4 \% 82.5 \%)$ [17] than the present study and the higher figure for skipping breakfast in Ankara was attributed to preferences to lose weight because overweight/obesity is their major concern.

In this study, age, sex, year of study, maternal education, father education, monthly income and resident area were not associated with underweight. The only attributes identified for underweight was using the campus Cafeteria, and eating place of non- café users which remained significant after controlling the confounding effect of socio-demographic factors. However some caution should be exercised in generalizing these findings because only ten percent of the students were assessed and significant differences in sociodemographic characteristics of those not captured in the study may have contributed to selection bias of the results. Thus a larger sample size to control for loss of underrepresented socio-demographic groups is recommended to determine the applicability of these findings.

On the other hand, eating outside of the university cafeteria was positively associated with the prevalence of overweight probably due to the fact that attending universitycould be stressful experience for many students and as aresult they tend to eat as a compensatory mechanism to get relief from their stress $[18,19]$.

Regarding the eating attitude of students, the study revealed that the mean eating attitude factors in the underweight, normal weight, overweight and obese students appear to be similar for most of the item factors. Some of the item factors of eating attitude observed to have opposite effects in subjects whose weight are normal and abnormal which is concordant with the Nigerian study [13]. It is interesting to see out of the 36 item factors assessed, six of them (like learning to cook, eating precooked/fast food, eating at the same time every day, intention to lose weight by consuming balanced diet and intention to strengthen relationships with others through eating together)had significant association with the level of overweight/obesity and underweight.

Although eating food when tastes good and eating more than usual (item 29) and practicing healthy eating behavior (item 7) which could lead to either weight gain, unexpectedly there were students who were underweight despite their food consumption. This observation may not reveal the true representation of the eating habits and BMI correlates among the studied population and requires further exploratory study.

Other interesting findings of this study were the correlates of overweight among students who mentioned seriously to learn cooking (item 2), consume more food when moody (item 6) and the food tastes (item 29), worry about calories (item 30), Fast food (item 11), eat slowly (item 13) and change their meals depending on their mood (item 14). This was expected as the consumption of excess food would lead to weight gain in most circumstances.

\section{Strength of the Study}

This study is first of its kind and had generated evidence 
based information for the institution to establish or strength the eating behaviors in relation to BMI status of students in university campus. In addition the reliability of the data was maintained by prior training for data collectors and regular supervision by principal investigator and using pretest questionnaire. Nonetheless, it was not easy to measure the temporal relationship since both exposure and outcome variables were collected simultaneously, and the study was limited to the three campuses and requires some caution in generalizing these findings. Additionally, self reported responses are highly dependent on the participant's memory, honesty and truthfulness in answering the questions lending some desirability biases. The correlates of BMI with psychosocial behavior is of the students is skipped in this article because it is too much and will be submitted soon as a separate article.

\section{Conclusion}

The study unveiled the presence of underweight and skipping breakfast among the participants. In addition, the mean eating attitude factors assessed among both students with normal and abnormal BMI status for most of the item factors were similar except for six of them. The predictors identified for underweight was using the campus Cafeteria, and eating place of non- café users. Nonetheless, this assertion requires some caution in generalizing these findings. To improve the prevailing nutritional problem, a coordinated effort at all level (family, university, community) and government is needed. A simple healthy eating guideline for the students might be a useful scheme for promoting such change, during their studies in university.

\section{Author's Contributions}

TTN and JHA conceptualized the study, wrote the manuscript, and approved the final version.

\section{Acknowledgment}

We thank Institute of Technology, Addis Ababa University for the financial support and the university management including all participants involved in the undertakings of the study.

\section{References}

[1] Walid E, Christiana S and Rafael T. Relationships between food consumption and living arrangements among university students in four European countries-A cross -sectional study. Nutrition journal 2012; 11:1-7.

[2] Nelson, M. C., Story, M., Larson, N. I., Neumark-Sztainer, D., \& Lytle, L. A.Emerging adulthood and college-aged youth: an overlooked age for weight-relatedbehavior change. Obesity 2008; 16:2205-2211.

[3] Savige GS, Ball K, Worsley A and Crawford D: Food intake patterns among Australian adolescents. Asia Pac J Clin Nutr 2007; 16: 738-747.

[4] Shi Z, Lien N, Kumar BN and Holmboe-Ottesen G: Sociodemographic differences in food habits patterns of school children and adolescents in and preferences of school adolescents in Jiangsu Province, China. Eur J Clin Nutr 2005, 59: $1439-1448$.

[5] Tom D, Peter C, Ilse D and Benedicte D. Determinants of Eating Behavior in University Students: A qualitative study using focus group discussions. BMC Public Health 2014; 14: 53 .

[6] Lua P, Wan D, Wan P, andSharhri L. Nutrition quality of life among female- majority Malay undergraduate students of Health Sciences 2012; 1: 1-7.

[7] Miso K and HongmieL. Overestimation of own body Weights in Female University Students: Associations with Life Styles, Weight control behaviors and depression. Nutrition Research and Practice 2010; 4(6): 499-506.

[8] La Caille, L. J., Dauner, K. N., Krambeer, R. J., \& Pedersen, J. Psychosocial and environmental determinants of eating behaviors, physical activity and weight change among college students: a qualitative analysis. Journal of American College Health 2011;59(6): 531-538.

[9] Gan W, Nasir M, Zalilah M and Haziza A. Difference in eating behaviors, dietary intake and body weight status between male and female Malaysian university students 2011; 17: 213-228.

[10] Addis Ababa University, Dean of Student Office summer of students registered for accadamic calender of 2014; 1: 1-10.

[11] Emanu A, Azeb A, Mezgebu Y and Kedir Y. Prevalence of overweight or obesity and associated factor among high school adolescents in Arada Sub city, Addis Ababa Ethiopia. J. Nutr Food sci 2014; 4:15.

[12] Takele T and Henock A. Hypertension and Associated factors among University Students in Gander, Ethiopia: acrosssectional study. BMC Public Health 2014;14: 1-5.

[13] Judith N, Kehi Hand Jephtha Christopher. Does eating behaviors among university students in Nigeria differ based on body mass index differences? Science Journal of Public Health 2014; 2(1): 38-46.

[14] Salman Z and Debore M. High rate of obesity-associated Hypertension amongprimary school children in Sudan. International Journal of Hypertension 2011; 2011: 1-5.

[15] Silliman, K., Rodas-Fortier, K., \& Neyman, M. A survey of dietary andexercise habits and perceived barriers to following a healthy lifestyle in a college population. Californian Journal of Health Promotion 2004; 2(2): 10-19.

[16] Anchamo A and Markos B. Assessment of breakfast eating habits and its association with cognitive performance of early adolescents (11-13 years) in Shebedino District, Sidama Zone, Southern Ethiopia. Journal of Food and Nutrition Sciences 2014; 2(4): 130-137.

[17] Ergulen S, Saygum M, Col M and Sayan M. A studyon theanemia, frequency, effectingfactors and eating habits among the students at Ankara University. JNut Diet 2001; 30: 24-31. 
[18] Gower B,and Crooks Z: The relationship between stress and eating in college-aged students. Undergraduate Research Journal for the human Science 2008; 7: 1-11.
[19] Santamaria A. R, Vazquez I. A, Caballero DP, Rodriguez CF. Eating habits and attitudes and their relationship with Body Mass Index (BMI). European Journal Psychiatric 2009; 23(4): 214-224. 